Sir,

\section{Management of Rhodotorula scleritis}

Infectious scleritis should be suspected in all cases of scleral inflammation following trauma. ${ }^{1,2}$ We report a case of microbial scleritis owing to the fungus Rhodotorula spp. and discuss its treatment.

\section{Case report}

A 40-year-old Asian-Indian lady complained of pain, redness, and a swelling on the left eye for 15 days preceded by trauma. Vision in both eyes was 6/6. Right eye was unremarkable. The left eye had a congested, non-ulcerated, scleral nodule measuring $5 \mathrm{~mm}$ in diameter on the temporal aspect of the limbus (Figure 1a). There was no keratitis, uveitis, or posterior segment abnormality. Review of systems was normal. Exploration revealed a tense intrascleral abscess that was excised en-mass. The pus was aspirated carefully to prevent contamination and sent for culture. There was no foreign body. Culture showed the presence of yeast that
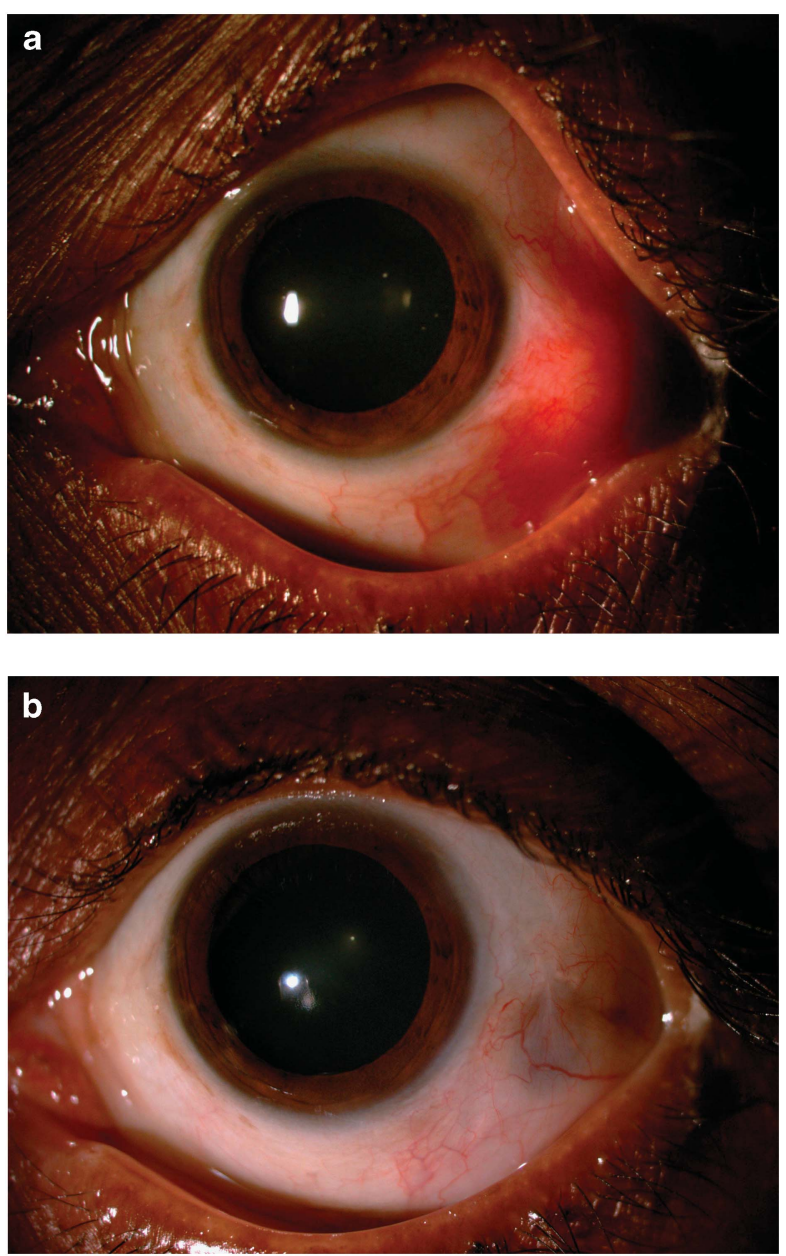

Figure 1 (a) Scleral abscess owing to Rhodotorula spp. (b) Healed scleritis with minimal scleral thinning. was identified as Rhodotorula spp. Topical amphotericin B $0.15 \%$ was instilled hourly. A boggy swelling reappeared 4 days later for which she underwent repeat surgical debridement under topical anaesthesia and irrigation with amphotericin. She responded well and amphotericin drops were tapered over 1 month. She did not have any recurrence of inflammation over the next 6 months and maintained a vision of $6 / 6$ with minimal scleral thinning (Figure 1b).

\section{Comment}

Although ocular fungal infections are not uncommon in the tropics, they are usually caused by filamentous fungi. ${ }^{1}$ Rhodotorula is a yeast of the family Cryptococcaceae that has been identified as a conjunctival commensal. ${ }^{3}$ It has been implicated in canaliculitis, endophthalmitis, and keratitis. ${ }^{4,5}$ We have not encountered any report of it causing scleritis.

Fungal scleritis is usually managed with debridement and prolonged topical and systemic antifungal medication. 2,5 The antifungal drug of choice for Rhodotorula is either flucytosine or amphotericin B. ${ }^{4}$ Flucytosine cannot be used alone owing to rapid development of resistance. ${ }^{5}$ Intravenous amphotericin B is effective but can cause nephrotoxicity, anaemia, and thrombophlebitis. ${ }^{5}$ Also, cases of Rhodotorula keratitis responded poorly to antifungals and required surgical intervention. ${ }^{5}$ Therefore, we chose to perform repeated debridements to decrease the organism load along with topical amphotericin to eradicate the infection that resulted in a favourable outcome without systemic side effects.

\section{Conflict of interest}

The authors declare no conflict of interest.

\section{References}

1 Jain V, Garg P, Sharma S. Microbial scleritis - experience from a developing country. Eye 2009; 23: 255-261.

2 Huang FC, Huang SP, Tseng SH. Management of infectious scleritis after pterygium excision. Cornea 2000; 19(1): 34-39.

3 Williamson J, Gordon AM, Wood R, Dyer AM, Yahya OA. Fungal flora of the conjunctival sac in health and disease influence of topical and systemic steroids. $\mathrm{Br}$ J Ophthalmol 1968; 52: 127-137.

4 Tuon FF, Costa. SF. Rhodotorula infection. A systematic review of 128 cases from literature. Rev Iberoam Micol 2008; 25: 135-140.

5 Thomas PA. Current perspectives on ophthalmic mycoses. Clin Microbiol Rev 2003; 16(4): 730-797.

ZS Pradhan and P Jacob

Department of Ophthalmology, Christian Medical College, Schell Eye Hospital, Vellore, India

E-mail: zedpradhan@gmail.com

Eye (2012) 26, 1587; doi:10.1038/eye.2012.181; published online 14 September 2012 